\title{
Dimensionamento amostral para avaliação de altura e diâmetro de mudas de Cabralea canjerana
}

\author{
Sample size for evaluating the height and diameter of seedlings Cabralea canjerana
}

\author{
Alberto Cargnelutti Filho ${ }^{I}$ Maristela Machado Araujo ${ }^{\text {II }}$ Ezequiel Gasparin"II \\ Angela Luciana de Avila ${ }^{\mathrm{IV}}$
}

\section{RESUMO}

Em viveiros florestais, é importante determinar o número de plantas a serem avaliadas para que as inferências sobre crescimento das mudas sejam confiáveis. O objetivo deste trabalho foi estimar o tamanho de amostra (número de plantas) necessário para avaliar a altura de planta, o diâmetro do coleto e a relação altura de planta/diâmetro do coleto de mudas de canjerana (Cabralea canjerana (Vell.) Mart.) $e$ verificar a variabilidade do tamanho de amostra entre tubetes, substratos e idades de avaliação. Foi conduzido um experimento, em viveiro, no qual foram avaliados nove tratamentos, formados pela combinação de três tamanhos de tubetes $\left(100,180\right.$ e $\left.280 \mathrm{~cm}^{3}\right)$ e três tipos de substratos $(100 \%$ de turfa, $80 \%$ de turfa $+20 \%$ de casca de arroz carbonizada e $60 \%$ de turfa $+40 \%$ de casca de arroz carbonizada), no delineamento experimental em blocos ao acaso, com quatro repetições. Em dez idades de avaliação, foram mensurados os caracteres altura de planta e diâmetro do coleto, e calculada a relação altura de planta/diâmetro do coleto, em dez plantas, tomadas aleatoriamente, na área central de cada unidade experimental. Foram realizadas análises de variância, testes de comparação de médias, verificação de normalidade e calculado o tamanho de amostra. O tamanho de amostra para avaliar a altura de planta, o diâmetro do coleto e a relação altura de planta/diâmetro do coleto de mudas de canjerana é dependente da idade de avaliação. Para os tubetes, substratos e idades de avaliação estudados, 18 plantas por unidade experimental (bandeja) são suficientes para a estimação da média desses caracteres, para um erro de estimação igual a $10 \%$ da média estimada, com grau de confiança de $95 \%$.

Palavras-chave: Canjerana, planejamento experimental, amostragem, precisão experimental.

\begin{abstract}
In nurseries, it is important to determine the number of plants to be evaluated so that inferences about seedling growth are reliable. The aim of this research was to estimate the sample size (number of plants) necessary to evaluate plant height, collar diameter and relation plant height/collar diameter of canjerana seedlings (Cabralea canjerana (Vell.) Mart.) and to verify its variability among tubes, substrates and ages evaluation. An experiment was conducted in the nursery, in which nine treatments, organized in randomizes blocks design with four replication, were formed by the combination of three sizes of tubes $\left(100,180\right.$ and $\left.280 \mathrm{~cm}^{3}\right)$ and three substrates (100\% peat, $80 \%$ peat $+20 \%$ rice hulls and $60 \%$ peat $+40 \%$ rice hulls). It was evaluated the following characters, in ten ages: plant height, collar diameter and relation plant height/ collar diameter, in ten plants sampled at random in each experimental unit. Analyses of variance, means comparison tests and verification of normality were carried and the sample size was calculated. The sample size to evaluate the plant height, collar diameter and relation plant height/collar diameter of canjerana seedlings is dependent on age evaluation. For the tubes, substrates and ages evaluation studied, 18 plants per experimental unit (pot) are enough to predict the these characters average, with an estimation error equal to $10 \%$ of estimated average, with a degree confidence of $95 \%$.
\end{abstract}

Key words: Canjerana, experimental planning, sampling, experimental precision.

\section{INTRODUÇÃO}

A Cabralea canjerana (Vell.) Mart. (canjerana) pertence à família Meliaceae. É considerada

'Departamento de Fitotecnia, Centro de Ciências Rurais (CCR), Universidade Federal de Santa Maria (UFSM), 97105-900, Santa

Maria, RS, Brasil. E-mail: alberto.cargnelutti.filho@gmail.com. Autor para correspondência.

"Departamento de Ciências Florestais, CCR, UFSM, Santa Maria, RS, Brasil.

IIIPrograma de Pós-graduação em Engenharia Florestal, UFSM, Santa Maria, RS, Brasil.

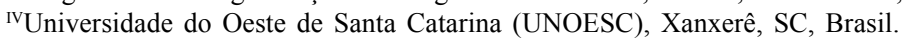


uma das espécies madeireiras mais valiosas do Brasil devido a sua madeira ser resistente às intempéries, com extraordinária durabilidade e com elevado valor econômico (BACKES \& IRGANG, 2009). Para o estabelecimento de plantios florestais, seja para fins comerciais ou para a restauração, é importante ter mudas de qualidade adequada. Para a produção de mudas em viveiros florestais, têm sido utilizados tubetes de diferentes tamanhos e preenchidos com diferentes tipos de substratos. Na formação do substrato, é comum misturar um comercial com outro material, como, por exemplo, a casca de arroz carbonizada.

Em experimentos conduzidos em viveiro florestal, é importante avaliar, com precisão desejada pelo pesquisador, caracteres como a altura de planta, $o$ diâmetro do coleto e, consequentemente, a relação altura de planta/diâmetro do coleto, durante o crescimento das plantas. A avaliação da qualidade morfológica de mudas florestais é importante para o sucesso no estabelecimento de plantios a campo. Mudas que apresentam altura inferior a $25 \mathrm{~cm}$ e diâmetro do coleto menor que $3 \mathrm{~mm}$ são, por exemplo, facilmente danificadas por formigas ou por chuvas torrenciais (DAVIDE \& FARIA, 2008). O valor da divisão desses dois caracteres, denominado também de quociente de robustez, representa o equilíbrio de crescimento, fornecendo informações de quão delgada está a muda (GOMES \& PAIVA, 2004). O diâmetro do coleto é a variável que melhor prediz o desempenho no pósplantio (RITCHIE et al., 2010), de modo que valores superiores desse caractere geralmente estão relacionados a um sistema radicular abundante, o que favorece o estabelecimento e o crescimento das plantas em condições de mato-competição. Nesse contexto, é importante dimensionar o número de plantas que devem ser avaliadas para possibilitar inferências precisas sobre esses caracteres.

A relação entre os erros experimental (variação entre as parcelas) e amostral (variação entre as plantas dentro da parcela) permite inferir quanto à adequabilidade dos aumentos do número de repetições ou do tamanho de amostra para melhoria da precisão experimental (BARBIN, 2003; RAMALHO et al., 2005; STORCK et al., 2006). Quando o erro experimental é significativamente superior ao erro amostral, o aumento do número de repetições do experimento é adequado. Caso contrário, pode-se melhorar a precisão experimental aumentando-se, também, o número de indivíduos por parcela (tamanho de amostra) (STORCK et al., 2006).

O tamanho de amostra ideal para mudas de Eucalyptus saligna, obtido em um estudo em viveiro florestal, com oito bandejas, cada uma contendo 96 plantas, foi de 26 mudas por tratamento, para uma diferença mínima significativa de $10 \%$, e de nove mudas por tratamento, quando a diferença foi de $20 \%$ para a variável diâmetro do coleto. Para a variável altura de planta, o tamanho ideal foi de 23 mudas para uma diferença de $10 \%$ e de oito mudas para diferença de $20 \%$ (ZANON et al., 1997). Para as variáveis diâmetro do coleto e altura, o tamanho de amostra para mudas de Pinus taeda L., em viveiro florestal, variou em função da idade das mudas, que foram avaliadas de $15 \mathrm{em} 15$ dias (SILVEIRA et al., 2009). Esses mesmos autores determinaram que o tamanho de amostra ideal para uma semiamplitude de $10 \%$ foi de 25 mudas para a altura e de 12 mudas para o diâmetro do coleto.

Não foram encontrados na literatura estudos sobre o dimensionamento de amostra para a avaliação de mudas de canjerana. Assim, o objetivo deste trabalho foi estimar o tamanho de amostra (número de plantas) necessário para avaliar a altura de planta, o diâmetro do coleto e a relação altura de planta/ diâmetro do coleto de mudas de canjerana e verificar a variabilidade do tamanho de amostra entre tubetes, substratos e idades de avaliação.

\section{MATERIAL E MÉTODOS}

Foi conduzido um experimento em casa de sombra coberta com tela de nylon 50\% com mudas de canjerana (Cabralea canjerana (Vell.) Mart.). Nesse experimento, foram avaliados nove tratamentos, formados pela combinação de três tamanhos de tubetes $\left(100,180\right.$ e $\left.280 \mathrm{~cm}^{3}\right)$ e três tipos de substratos $(100 \%$ de turfa, $80 \%$ de turfa $+20 \%$ de casca de arroz carbonizada e $60 \%$ de turfa $+40 \%$ de casca de arroz carbonizada). $O$ delineamento experimental foi em blocos ao acaso, com quatro repetições. As 36 unidades experimentais (parcelas) foram constituídas por bandejas de polipropileno de $43,5 \mathrm{~cm}$ de largura $\mathrm{x} 63,0 \mathrm{~cm}$ de comprimento e $16,5 \mathrm{~cm}$ de altura. Nas bandejas com os tubetes de $100 \mathrm{~cm}^{3}$, foram colocados 96 tubetes e, nas bandejas com os tubetes de 180 e $280 \mathrm{~cm}^{3}$, foram colocados 54 tubetes, totalizando 2.448 tubetes no experimento.

Em janeiro de 2009, foram coletados frutos de canjerana de cinco árvores matrizes, na localidade de Santo Antão, Santa Maria, Rio Grande do Sul. Após, foram beneficiados os frutos, deixando-os dentro de sacos de polietileno fechados por uma semana, em local sombreado e ventilado para promover a deiscência e liberação das sementes. Em seguida, as sementes foram lavadas para a extração do arilo e secas em ambiente sombreado. Em 05 de fevereiro de 2009, foram semeadas duas sementes em cada tubete de cada parcela, 
ajustando-se, aos 30 dias após a semeadura, para uma planta por tubete, por meio de raleio.

A adubação de base foi constituída de ureia, superfosfato simples e cloreto de potássio, nas dosagens de $350,4.000$ e $200 \mathrm{~g} \mathrm{~m}^{-3}$ de substrato, respectivamente. A partir dos 60 dias após a semeadura, foram realizadas, semanalmente, adubações de cobertura com o adubo Peter's Professional ${ }^{\circledR}(9-45-15)$ na dosagem de $3 \mathrm{~g} \mathrm{~L}^{-1}$, aplicado via solução aquosa por meio de pulverizador costal.

Aos 60, 90, 120, 150, 180, 210, 240, 270, 300,

330 dias após a semeadura (DAS), foi mensurada a altura de planta $(\mathrm{H})$, em centímetros, com régua milimetrada e o diâmetro do coleto (D), em milímetros, com paquímetro digital, e calculada a relação altura de planta/diâmetro do coleto (HD), em dez plantas, tomadas aleatoriamente na área central de cada parcela. Nas dez idades, sempre foram avaliadas as mesmas dez plantas de cada parcela.

Os dados dos caracteres H, D e HD, em cada uma das dez idades de avaliação, foram submetidos à análise de variância e teste $\mathrm{F}$ a $5 \%$ de probabilidade. As pressuposições de normalidade dos erros e homogeneidade das variâncias residuais foram atendidas. Para a análise de variância, foi utilizado o modelo matemático do delineamento em blocos ao acaso com amostragem nas parcelas, definido em STORCK et al. (2006).

A seguir, para os dados de H, D e HD das dez plantas de cada uma das 36 parcelas, nas dez idades de avaliação, foi verificada a normalidade, por meio do teste de Kolmogorov-Smirnov (CAMPOS, 1983; SIEGEL \& CASTELLAN JÚNIOR, 2006), totalizando 1.080 testes ( 36 parcelas/avaliação $\times 10$ avaliações $\times 3$ caracteres). A normalidade foi investigada a fim de verificar a adequação desses dados para o estudo do dimensionamento amostral, com base na distribuição $t$ de Student.

Para os caracteres H, D e HD, tomando-se por base as dez plantas amostradas em cada unidade experimental (parcela), de cada combinação de tamanho de tubete e tipo de substrato, em cada idade de avaliação, foi calculado o tamanho de amostra (número de plantas, $\eta$ ) para as semiamplitudes do intervalo de confiança (erro de estimação) iguais a 1,2, ..., 10\% da estimativa da média (m), com grau de confiança $(1-\alpha)$

de $95 \%$, por meio da expressão $\quad \eta=\frac{\mathrm{t}_{\alpha / 2}^{2} \mathrm{~s}^{2}}{(\text { erro de estimação })^{2}}$

(BARBETTA et al., 2004; BUSSAB \& MORETTIN, 2004; SPIEGEL et al., 2004), na qual t $\alpha: 2$ é o valor crítico da distribuição t de Student, cuja área à direita é igual a $\alpha / 2$, isto é, o valor de $t$, tal que $\mathrm{P}\left(\mathrm{t}>\mathrm{t} \alpha_{/ 2}\right)=\alpha / 2$, com (n-
1) graus de liberdade ( $n=10$ plantas $)$, com $\alpha=5 \%$ de probabilidade de erro, e s ${ }^{2}$ é a estimativa de variância amostral. Assim, obtiveram-se 30 variáveis (tamanho de amostra) formadas pela combinação dos caracteres H, D e HD com os níveis de precisão de 1, 2, 3, 4, 5, 6, 7, 8,9 e $10 \%$ da média estimada, na unidade experimental.

A fim de investigar a variabilidade do tamanho de amostra entre tubetes, substratos e idades de avaliação, os dados dessas 30 variáveis (tamanho de amostra) foram submetidos à análise de variância considerando o modelo matemático do delineamento em blocos ao acaso com parcelas subdivididas no tempo, definido em STORCK et al. (2006). Nesse modelo, os nove tratamentos (três tamanhos de tubetes $\times$ três tipos de substratos) e as dez idades de avaliação formaram, respectivamente, a parcela principal e a subparcela no tempo. As médias do efeito principal de idades de avaliação foram comparadas por meio do teste de Scott Knott (RAMALHO et al., 2005) a 5\% de probabilidade. As análises estatísticas foram realizadas com o auxílio do programa SISVAR (FERREIRA, 2008) e do aplicativo Office Excel.

\section{RESULTADOS E DISCUSSÃO}

Nas dez idades de avaliação, não houve efeito significativo da interação tubete $\times$ substrato em relação aos caracteres altura de planta $(\mathrm{H})$, diâmetro do coleto (D) e relação altura de planta/diâmetro do coleto (HD), indicando que esses fatores são independentes, ou seja, para esses caracteres, as diferenças entre as plantas nos tubetes não variam com os substratos e vice-versa. $\mathrm{O}$ efeito principal de tubete foi significativo em relação à $\mathrm{H}$, ao D e à $\mathrm{HD}$, em $40 \%, 70 \%$ e $100 \%$ das idades de avaliação, respectivamente, o que evidencia que há variabilidade entre as plantas nos diferentes tamanhos de tubetes. Por sua vez, o efeito principal de substrato foi significativo em relação à $\mathrm{H}$, ao $\mathrm{D}$ e à $\mathrm{HD}$, em $90 \%$, $80 \%$ e $10 \%$ das idades de avaliação, respectivamente, o que revela que nesses substratos as plantas apresentam diferenças em relação a esses três caracteres (Tabela 1). Testes de comparações múltiplas de médias, dos efeitos principais de tubetes e de substratos, seriam adequados para a identificação do tamanho de tubete e do tipo de substrato que proporcionariam mudas com desempenho superior em relação à $\mathrm{H}$, ao $\mathrm{D}$ e à $\mathrm{HD}$, até os 330 dias após a semeadura, mas não realizados por não ser o foco deste trabalho.

O erro experimental (variação entre as parcelas) foi significativo em $100 \%, 50 \%$ e $10 \%$ das idades de avaliação, respectivamente, em relação aos caracteres H, D e HD (Tabela 1). Isso indica que nesses 16 casos o aumento do número de repetições é 
Tabela 1 - Número de graus de liberdade (GL) e quadrado médio para as fontes de variação bloco, tubete, substrato, tubete× substrato, erro experimental e erro amostral, média, coeficiente de variação experimental (CVe) e coeficiente de variação amostral (CVa), para três caracteres de mudas de canjerana (Cabralea canjerana) avaliados em nove tratamentos (três tamanhos de tubetes $\times$ três tipos de substratos) durante dez idades de avaliação.

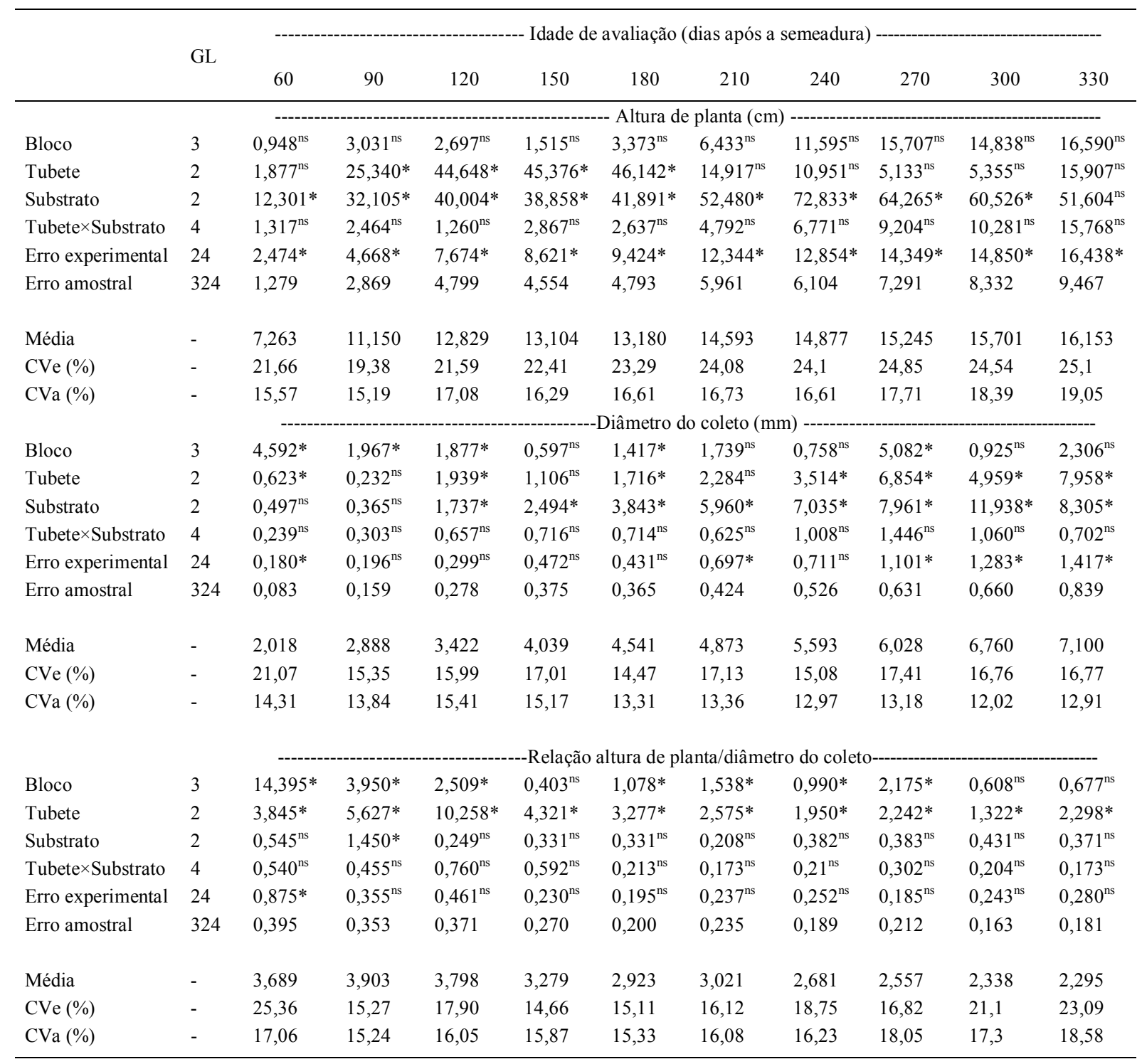

*Efeito significativo pelo teste $\mathrm{F}$ em nível de $5 \%$ de probabilidade. ${ }^{\text {ns }}$ Não significativo.

apropriado para melhorar a precisão experimental (BARBIN, 2003; STORCK et al., 2006). Por outro lado, nos demais 14 casos, o efeito do erro experimental não foi significativo, revelando que o aumento do tamanho de amostra é adequado para a melhoria da precisão experimental (STORCK et al., 2006). Portanto, o redimensionamento do uso de quatro repetições (blocos) e a amostragem de dez plantas por parcela para avaliar os caracteres H, D e HD são convenientes para melhorar a precisão experimental.
Experimentos com coeficiente de variação experimental (CVe) inferior a $10 \%$, entre $10 \%$ e $20 \%$, entre $20 \%$ e $30 \%$ e superiores a $30 \%$ são classificados, respectivamente, como de alta, média, baixa e muito baixa precisão experimental por GOMES (1990). Embora essa classificação tenha sido proposta para experimentos a campo, ela tem sido extensivamente utilizada. Assim, entre os 30 casos (três caracteres $\times$ dez idades de avaliação), em 13 (43,3\%) e em 17 (56,7\%) casos, os $\mathrm{CVe}$ foram enquadrados, respectivamente, 
nas faixas de baixa e média precisão experimental. Esses resultados confirmam as inferências anteriores de que o redimensionamento do planejamento experimental é um procedimento adequado a fim de obter experimentos com alta precisão experimental $(\mathrm{CVe}<10 \%)$.

O coeficiente de variação experimental (CVe) médio das dez idades de avaliação foi $23,10 \%, 16,70 \%$ e $18,42 \%$, respectivamente, para os caracteres H, D e HD. Já o coeficiente de variação amostral (CVa) médio das dez idades de avaliação foi 16,92\%, 13,65\% e $16,58 \%$, respectivamente, para os caracteres H, D e HD. Esses resultados evidenciam, de maneira geral, menor variabilidade dentro de unidades experimentais do que entre estas. Portanto, embora o aumento do tamanho de amostra seja eficiente, de maneira geral, aumentar o número de repetições é um procedimento adequado para melhorar a precisão experimental (BARBIN, 2003). Os caracteres $\mathrm{H}$ e HD apresentaram maiores valores médios de $\mathrm{CVa}$, o que comprova maior variabilidade entre as plantas dentro da parcela comparativamente ao $\mathrm{D}$, sugerindo que, em mudas de canjerana, para a estimação da média, com uma mesma precisão, o tamanho de amostra para o caractere D seja menor em relação à $\mathrm{H}$ e à $\mathrm{HD}$. Esse resultado pode estar associado ao fato de que o diâmetro é influenciado, principalmente, pelas condições do meio, as quais são homogêneas em experimentos conduzidos em viveiro, enquanto a altura está mais associada a fatores genéticos (KRAMER \& KOZLOWSKI, 1972), os quais têm maior dependência do material de origem. Assim, considerando que as sementes utilizadas foram provenientes de material silvestre, essa maior variação pode ser explicada.

O valor de $\mathrm{P}$ (nível mínimo de significância) do teste de Kolmogorov-Smirnov (CAMPOS, 1983; SIEGEL \& CASTELLAN JÚNIOR, 2006), em relação aos dados das dez plantas, dos 1.080 casos analisados (36 parcelas/avaliação $\times 10$ avaliações $\times 3$ caracteres), oscilou entre 0,127 e 0,999, e a média foi de 0,829. Esses resultados evidenciam boa aderência dos dados à distribuição normal $(\mathrm{P}>0,126)$ e, portanto, são adequados para o estudo do dimensionamento amostral, com base na distribuição t de Student.

Em relação ao tamanho de amostra para a estimação da média dos caracteres $\mathrm{H}, \mathrm{D}$ e HD, não houve efeitos significativos da interação tripla (Tubete $\times$ Substrato $\times$ Idade) e das interações duplas (Tubete $\times$ Substrato, Tubete $\times$ Idade e Substrato $\times$ Idade) (Tabela 2). Isso indica que há independência desses fatores em relação ao tamanho de amostra. Os efeitos principais de tubete e de substrato também não foram significativos, o que evidencia que não há variabilidade

Tabela 2 - Número de graus de liberdade (GL), valor calculado do teste F (F) e p-valor para as fontes de variação bloco, tubete, substrato, tubete $\times$ substrato, erro 1 (bloco $\times$ tratamento), idade, erro $_{2}$ (bloco $\times$ idade), tubete $\times$ idade, substrato $\times$ idade, tubete $\times$ substrato $\times$ idade $\mathrm{e}$ erro $_{3}$ (bloco×tratamento $\times$ idade) e coeficientes de variação relacionados aos erros $1\left(\mathrm{CV}_{1}\right), 2\left(\mathrm{CV}_{2}\right)$ e $3\left(\mathrm{CV}_{3}\right)$, para os tamanhos de amostra de três caracteres ${ }^{(1)}$ de mudas de canjerana (Cabralea canjerana) avaliados em nove tratamentos (três tamanhos de tubetes $\times$ três tipos de substratos) e em dez idades de avaliação.

\begin{tabular}{|c|c|c|c|c|c|c|c|}
\hline & $C T$ & $\mathrm{~F}$ & $\mathrm{p}$-valor & $\mathrm{F}$ & p-valor & $\mathrm{F}$ & p-valor \\
\hline Bloco & 3 & 4,836 & 0,009 & 0,767 & 0,524 & 0,329 & 0,804 \\
\hline Tubete & 2 & 1,088 & 0,353 & 1,879 & 0,175 & 0,792 & 0,465 \\
\hline Substrato & 2 & 0,822 & 0,452 & 0,714 & 0,500 & 0,215 & 0,808 \\
\hline Tubete $\times$ Substrato & 4 & 0,191 & 0,941 & 1,492 & 0,236 & 0,092 & 0,984 \\
\hline Erro $_{1}($ Bloco $\times$ Tratamento $)$ & 24 & - & - & - & - & - & - \\
\hline Idade & 9 & 7,125 & 0,000 & 2,269 & 0,048 & 5,159 & 0,000 \\
\hline Erro $_{2}($ Bloco $\times$ Idade $)$ & 27 & - & - & - & - & - & - \\
\hline Tubete $\times$ Idade & 18 & 0,515 & 0,950 & 1,580 & 0,067 & 0,628 & 0,875 \\
\hline Substrato $\times$ Idade & 18 & 0,850 & 0,639 & 0,907 & 0,571 & 0,236 & 0,999 \\
\hline Tubete $\times$ Substrato $\times$ Idade & 36 & 0,306 & 0,999 & 1,356 & 0,097 & 0,725 & 0,875 \\
\hline Erro $_{3}($ Bloco $\times$ Tratamento $\times$ Idade $)$ & 216 & - & - & - & - & - & - \\
\hline $\mathrm{CV}_{1}(\%)$ & - & 107,83 & & 101,39 & & 96,24 & \\
\hline $\mathrm{CV}_{2}(\%)$ & - & 31,98 & & 62,52 & & 34,84 & \\
\hline $\mathrm{CV}_{3}(\%)$ & - & 34,76 & & 41,39 & & 42,60 & \\
\hline
\end{tabular}

${ }^{(1)} \mathrm{H}=$ tamanho de amostra para a altura de planta, $\mathrm{D}=$ tamanho de amostra para o diâmetro do coleto e HD = tamanho de amostra para a relação altura de planta/diâmetro do coleto. * Efeito significativo pelo teste $\mathrm{F}$ em nível de $5 \%$ de probabilidade (aproximado). ${ }^{\text {ns }}$ Não significativo. 
do tamanho de amostra entre tubetes e entre substratos. Por sua vez, o efeito de idade de avaliação foi significativo $(\mathrm{P} \leq 0,05)$, o que revela que o tamanho de amostra para avaliar esses três caracteres é dependente da idade de avaliação. Em resumo, para a estimação da média dos caracteres $\mathrm{H}, \mathrm{D}$ e HD, com uma mesma precisão, o tamanho de amostra (número de plantas) é único para os diferentes tubetes e substratos e variável com as idades de avaliação. Para a avaliação do diâmetro do coleto e da altura de mudas de Pinus taeda L., SILVEIRA et al. (2009) também relataram que o tamanho de amostra variou em função da idade das mudas, devido ao crescimento heterogêneo das mudas com o passar das avaliações.

Quanto ao tamanho de amostra para a estimação da média dos caracteres H, D e HD, as idades de avaliação foram separadas em dois grupos por meio do teste de Scott Knott (Tabela 3). Em relação à H, as sete primeiras avaliações, ou seja, até os 240 dias após a semeadura, compuseram um grupo com menor variabilidade entre as plantas dentro da parcela, necessitando menor número de plantas para estimar a média com uma mesma precisão, quando comparadas as demais três idades de avaliação do outro grupo. Por outro lado, de maneira geral, o tamanho de amostra para a estimação da média de $\mathrm{D}$, apresentou comportamento contrário ao anterior, ou seja, com exceção da avaliação aos 90 dias de idade, as três primeiras avaliações (até 150 dias após a semeadura) compuseram o grupo com maior variabilidade entre as plantas dentro da parcela, necessitando maior número de plantas quando comparadas às demais idades de avaliação. Já, em relação ao caractere HD, maior tamanho de amostra foi verificado na primeira idade de avaliação (60 dias após a semeadura) e nas três últimas idades de avaliação. Portanto, de maneira geral, pode-se inferir que, para os caracteres $\mathrm{H}$ e HD, é necessário maior tamanho de amostra nas últimas avaliações. $\mathrm{O}$ maior tempo de atuação do ambiente sobre essas últimas avaliações pode, provavelmente, explicar a maior variabilidade dos caracteres H e HD. Aspectos relacionados ao material propagativo (seminal), ao esgotamento da fertilização de base, à quantidade de substrato e à restrição imposta pelos tubetes para o crescimento do sistema radicular, também podem interferir na variabilidade. Por outro lado, em relação ao $\mathrm{D}$, houve tendência de diminuição da variabilidade entre as plantas dentro da parcela com as idades de avaliação, o que refletiu em menor tamanho de amostra. Menor tamanho de amostra para a avaliação do diâmetro de coleto em relação à altura de mudas de Pinus taeda L., também foi observado por SILVEIRA et al. (2009).
O tamanho de amostra (número de plantas) para a estimação da média de cada caractere, com semiamplitude do intervalo de confiança igual a $1 \%$ da estimativa da média (m) e coeficiente de confiança de $95 \%$, oscilou de 740 plantas, para o caractere diâmetro do coleto avaliado aos 300 dias após a semeadura a 1.843 plantas, para a $\mathrm{H}$ aos 330 dias após a semeadura (Tabela 3). Portanto, em relação ao diâmetro do coleto avaliado aos 300 dias após a semeadura, pode-se inferir, com $95 \%$ de confiança, que o intervalo de confiança da média $(\mu)$, obtida com 740 plantas, é de $\mu \pm 0,01 \mathrm{~m}$. Em outro extremo, a precisão de $\mu \pm 0,01 \mathrm{~m}$ é obtida com 1.843 plantas, para a altura de planta aos 330 dias após a semeadura. Esses resultados confirmam a variabilidade existente entre as idades de avaliação e entre os caracteres. Portanto, de maneira geral, os resultados evidenciam que, do ponto de vista de técnicas experimentais, ao dimensionar o tamanho de amostra para uma avaliação precisa de mudas de canjerana, os caracteres e a idade das plantas devem ser considerados.

Particularmente, neste experimento, o número máximo de plantas por parcela foi 96 . Então, estimativas com precisão inferior a 5\%, de modo geral, são impraticáveis. Assim, menores tamanhos de amostra foram determinados com base em semiamplitudes do intervalo de confiança iguais a 6,7, 8,9 e $10 \%$ da média estimada (Tabela 3 ). Não cabe aqui o julgamento do erro de estimação máximo aceitável, ficando essa tarefa ao usuário dessas informações, de acordo com a disponibilidade de dados e a precisão desejada. Portanto, tomando-se como referência o maior tamanho de amostra (altura de planta avaliada aos 330 dias após a semeadura), pode-se inferir, com $95 \%$ de confiança, que, com 18 plantas, o erro máximo na estimativa da média (m) de H, D e HD será de $\pm 10 \%$ da média estimada, independentemente do tubete e substrato.

\section{CONCLUSÃO}

O tamanho de amostra (número de plantas) para avaliar a altura de planta, o diâmetro do coleto e a relação altura de planta/diâmetro do coleto de mudas de canjerana (Cabralea canjerana) é dependente da idade de avaliação. Para os tamanhos de tubetes, tipos de substratos e idades de avaliação estudados, 18 plantas por unidade experimental são suficientes para estimar a média de altura de planta, de diâmetro do coleto e de relação altura de planta/diâmetro do coleto de mudas de canjerana, para um erro de estimação igual a $10 \%$ da média estimada, com grau de confiança de $95 \%$. 
Tabela 3 - Tamanho de amostra (número de plantas) para avaliação dos caracteres altura de planta, diâmetro do coleto e relação altura de planta/diâmetro do coleto de mudas de canjerana (Cabralea canjerana), para as semiamplitudes do intervalo de confiança de 95\% (erro de estimação) iguais a 1,2, .., 10\% da estimativa da média, em dez idades de avaliação (Idade), em dias após a semeadura.

\begin{tabular}{|c|c|c|c|c|c|c|c|c|c|c|}
\hline \multirow{2}{*}{ Idade } & \multicolumn{10}{|c|}{ 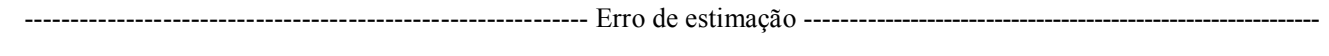 } \\
\hline & $1 \%$ & $2 \%$ & $3 \%$ & $4 \%$ & $5 \%$ & $6 \%$ & $7 \%$ & $8 \%$ & $9 \%$ & $10 \%$ \\
\hline \multicolumn{11}{|c|}{ 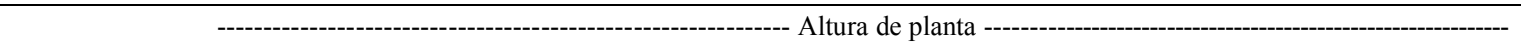 } \\
\hline 60 & 1.240 & 310 & 138 & 78 & 50 & 34 & 25 & 19 & 15 & $12 b^{*}$ \\
\hline 90 & 1.170 & 293 & 130 & 73 & 47 & 33 & 24 & 18 & 14 & $12 \mathrm{~b}$ \\
\hline 120 & 1.464 & 366 & 163 & 92 & 59 & 41 & 30 & 23 & 18 & $15 \mathrm{~b}$ \\
\hline 150 & 1.346 & 337 & 150 & 84 & 54 & 37 & 27 & 21 & 17 & $13 \mathrm{~b}$ \\
\hline 180 & 1.386 & 346 & 154 & 87 & 55 & 38 & 28 & 22 & 17 & $14 \mathrm{~b}$ \\
\hline 210 & 1.426 & 357 & 158 & 89 & 57 & 40 & 29 & 22 & 18 & $14 \mathrm{~b}$ \\
\hline 240 & 1.403 & 351 & 156 & 88 & 56 & 39 & 29 & 22 & 17 & $14 \mathrm{~b}$ \\
\hline 270 & 1.603 & 401 & 178 & 100 & 64 & 45 & 33 & 25 & 20 & $16 \mathrm{a}$ \\
\hline 300 & 1.717 & 429 & 191 & 107 & 69 & 48 & 35 & 27 & 21 & $17 \mathrm{a}$ \\
\hline 330 & 1.843 & 461 & 205 & 115 & 74 & 51 & 38 & 29 & 23 & $18 \mathrm{a}$ \\
\hline \multicolumn{11}{|c|}{ 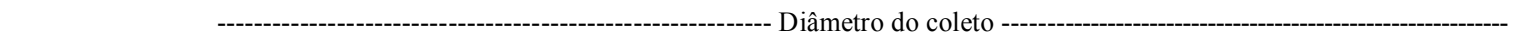 } \\
\hline 60 & 1.104 & 276 & 123 & 69 & 44 & 31 & 23 & 17 & 14 & $11 \mathrm{a}$ \\
\hline 90 & 982 & 246 & 109 & 61 & 39 & 27 & 20 & 15 & 12 & $10 \mathrm{~b}$ \\
\hline 120 & 1.210 & 303 & 134 & 76 & 48 & 34 & 25 & 19 & 15 & $12 \mathrm{a}$ \\
\hline 150 & 1.166 & 291 & 130 & 73 & 47 & 32 & 24 & 18 & 14 & $12 \mathrm{a}$ \\
\hline 180 & 906 & 227 & 101 & 57 & 36 & 25 & 18 & 14 & 11 & $9 \mathrm{~b}$ \\
\hline 210 & 920 & 230 & 102 & 57 & 37 & 26 & 19 & 14 & 11 & $9 \mathrm{~b}$ \\
\hline 240 & 858 & 214 & 95 & 54 & 34 & 24 & 18 & 13 & 11 & $9 \mathrm{~b}$ \\
\hline 270 & 885 & 221 & 98 & 55 & 35 & 25 & 18 & 14 & 11 & $9 \mathrm{~b}$ \\
\hline 300 & 740 & 185 & 82 & 46 & 30 & 21 & 15 & 12 & 9 & $7 \mathrm{~b}$ \\
\hline 330 & 859 & 215 & 95 & 54 & 34 & 24 & 18 & 13 & 11 & $9 \mathrm{~b}$ \\
\hline & & & & -- Rela & tura d & a/diân & o cole & ----- & 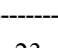 & ---- \\
\hline 60 & 1.489 & 372 & 165 & 93 & 60 & 41 & 30 & 18 & 23 & $15 \mathrm{a}$ \\
\hline 90 & 1.159 & 290 & 129 & 72 & 46 & 32 & 24 & 14 & 18 & $12 \mathrm{~b}$ \\
\hline 120 & 1.293 & 323 & 144 & 81 & 52 & 36 & 26 & 16 & 20 & $13 \mathrm{~b}$ \\
\hline 150 & 1.286 & 321 & 143 & 80 & 51 & 36 & 26 & 16 & 20 & $13 \mathrm{~b}$ \\
\hline 180 & 1.185 & 296 & 132 & 74 & 47 & 33 & 24 & 15 & 19 & $12 \mathrm{~b}$ \\
\hline 210 & 1.321 & 330 & 147 & 83 & 53 & 37 & 27 & 16 & 21 & $13 \mathrm{~b}$ \\
\hline 240 & 1.330 & 332 & 148 & 83 & 53 & 37 & 27 & 16 & 21 & $13 \mathrm{~b}$ \\
\hline 270 & 1.611 & 403 & 179 & 101 & 64 & 45 & 33 & 20 & 25 & $16 \mathrm{a}$ \\
\hline 300 & 1.494 & 373 & 166 & 93 & 60 & 41 & 30 & 18 & 23 & $15 \mathrm{a}$ \\
\hline 330 & 1.719 & 430 & 191 & 107 & 69 & 48 & 35 & 21 & 27 & $17 \mathrm{a}$ \\
\hline
\end{tabular}

*Médias não seguidas de mesma letra diferem pelo teste de Scott Knott em nível de 5\% de probabilidade (aproximado). Nas colunas referentes aos erros de estimação de 1 a $9 \%$, as letras são as mesmas da coluna referente ao erro de estimação de $10 \%$, e, por isso, não foram colocadas.

\section{AGRADECIMENTOS}

Ao Conselho Nacional de Desenvolvimento Científico e Tecnológico ( $\mathrm{CNPq})$, pela concessão de bolsa de Produtividade em Pesquisa para Alberto Cargnelutti Filho.

\section{REFERÊNCIAS}

BACKES, P.; IRGANG, B. Árvores do sul: guia de identificação e interesse ecológico. Porto Alegre: Paisagem do Sul, 2009. 332 p.

BARBETTA, P.A. et al. Estatística para cursos de engenharia e informática. São Paulo: Atlas, 2004. 410p.
BARBIN, D. Planejamento e análise estatística de experimentos agronômicos. Arapongas: Midas, 2003. 208p.

BUSSAB, W.O.; MORETTIN, P.A. Estatística básica. 5.ed. São Paulo: Saraiva, 2004. 526p.

CAMPOS, H. Estatística experimental não-paramétrica. 4.ed. Piracicaba: Departamento de Matemática e Estatística ESALQ, 1983. 349p.

DAVIDE, A.C.; FARIA, J.M.R. Viveiros florestais. In: DAVIDE, A.C.; SILVA, E.A.A. (Ed.). Produção de sementes e mudas de espécies florestais. Lavras: UFLA, 2008. Cap.2, p.83-124. 
FERREIRA, D.F. SISVAR: um programa para análises e ensino de estatística. Revista Symposium, v.6, p.36-41, 2008

GOMES, F.P. Curso de estatística experimental. 13.ed. Piracicaba: São Paulo: Nobel 1990. 468p.

GOMES, J.M.; PAIVA, H.N. Viveiros florestais (propagação sexuada). 3.ed. Viçosa: UFV, 2004. 116p. (Cadernos Didáticos, 72).

KRAMER, P.J.; KOZLOWSKI, T.T. Fisiologia das árvores Lisboa: Fundação Calouste Gulbenkian, 1972. 745p.

RAMALHO, M.A.P. et al. Experimentação em genética e melhoramento de plantas. Lavras: UFLA, 2005. 322p.

RITCHIE, G.A. et al. Assessing plant quality. In: LANDIS, T.D. et al. Seedling processing, storage and outplanting
Washington, DC: US Department of Agriculture Forest Service, 2010. V.7, cap.2, p.17-81. (Agriculture. Handbook. 674)

SIEGEL, S.; CASTELLAN JÚNIOR, N.J. Estatística nãoparamétrica para ciências do comportamento. 2.ed. Porto Alegre: Artmed, 2006. 448p.

SILVEIRA, B.D. et al. Aleatoriedade e tamanho da amostra em mudas de Pinus taeda L. Ceres, v.56, p.730-735, 2009.

SPIEGEL, M.R. et al. Probabilidade e estatística. 2.ed. Porto Alegre: Bookman, 2004. 398p.

STORCK, L. et al. Experimentação vegetal. 2.ed. Santa Maria: UFSM, 2006. 198p.

ZANON, M.L.B. et al. Tamanho de amostra para experimentos de Eucalyptus saligna SMITH em viveiro. Ciência Florestal v.7, p.133-138, 1997. 